\title{
Neurologic immune-related adverse events associated with adjuvant ipilimumab: report of two cases
}

\author{
Christine A. Garcia' ${ }^{1}$, Alex El-Ali², Tanya J. Rath² ${ }^{2}$ Lydia C. Contis ${ }^{3}$, Vikram Gorantla', Jan Drappatz \\ and Diwakar Davar ${ }^{*}$ (D)
}

\begin{abstract}
Background: PD-1 and CTLA-4 inhibitors are associated with several adverse events including a spectrum of immune-related adverse effects (irAEs). Neurologic irAEs are uncommon occurrences with varied presentations. We describe two separate cases of ipilimumab associated meningoencephalomyelitis and demyelinating polyneuropathy with unusual presentations.

Case presentation: Two melanoma patients were treated with ipilimumab in the adjuvant setting. The first patient developed a meningoencephalitis following 3 doses of ipilimumab. MRI imaging of the brain confirmed leptomeningeal enhancement although cerebrospinal fluid (CSF) analyses were negative for malignant cells consistent with meningoencephalomyelitis. Although she initially improved following treatment with steroids and intravenous immunoglobulin, she subsequently relapsed. She was successfully treated with infliximab and made a complete neurological recovery. A second patient developed progressive lower extremity weakness following two doses of ipilimumab. MRI imaging of the spine confirmed diffuse nerve root enhancement consistent with acute inflammatory demyelinating polyneuropathy (AIDP). He was treated with high dose steroids with resolution of neurological symptoms. Both patients remain disease free.

Conclusions: Neurological irAEs are uncommon adverse events in the context of CTLA-4 and/or PD-1 inhibitor therapy. Care must be taken to distinguish these from leptomeningeal disease. Early recognition of neurological irAEs is critical for the initiation of specific anti-inflammatory agents to prevent and potentially reverse neurological sequelae.
\end{abstract}

Keywords: Cancer, Melanoma, Immunotherapy, Ipilimumab, Infliximab, Immune-related adverse events, CTLA-4, TNF-a, Neurotoxicity, Autoimmune, Guillain Barre syndrome, Meningoencephalomyelitis

\section{Background}

Cytotoxic T lymphocyte antigen-4 (CTLA-4) and CD28 are homologues with diametric effects on T cell activation. While the B7/CD28 interaction provides the second "costimulatory" signal required for $\mathrm{T}$ cell activation; by cross-linking CD28 and T-cell receptor (TCR) CTLA-4 strongly inhibits T cell activation [1]. Consequently, inhibition of CTLA-4 by monoclonal antibodies such as ipilimumab and tremelimumab promotes anti-tumor immunity [2]. Ipilimumab is a fully

\footnotetext{
* Correspondence: davard@upmc.edu

'Hillman Cancer Center, University of Pittsburgh Medical Center, 5150 Centre Ave, Pittsburgh, PA 15232, USA

Full list of author information is available at the end of the article
}

humanized IgG1 monoclonal antibody that inhibits the CD28/CTLA-4 interaction, thereby promoting T cell activation [3] and resulting in anti-tumor immunity [4]. Ipilimumab therapy improves survival in metastatic melanoma and results in durable remissions with 3-year survival rates of $20 \%$ and $26 \%$ in treated and treatment-naïve melanoma patients respectively [5-7].

Ipilimumab is associated with several adverse events including a spectrum of immune-related adverse effects (irAEs) that include enterocolitis, pneumonitis, hepatitis, dermatitis, hypophysitis and nephritis [8]. Incidence of irAEs in phase II and III trials differ widely: dermatitis (47-68\%), enterocolitis (31-46\%), hepatitis (3-9\%), hypophysitis (4- 
$6 \%)$, pancreatitis (1.5\%), uveitis (1\%), lymphadenopathy (1\%) and neurologic events $(0.1 \%)$ [9]. The incidence of neurologic irAEs in ipilimumab phase II and III trials was $0.1 \%$ with no grade 3-4 events [10], although cases of nerve palsies, demyelination, limbic encephalitis, Guillain-Barre Syndrome (GBS) and myasthenia gravis have been reported [9]. The etiology of ipilimumab-induced irAEs is unclear; possible explanations include the loss of peripheral tolerance mediated by CTLA- 4 and ectopic expression of CTLA- 4 at least in the setting of hypophysitis [11].

Well-established algorithms for the management of neurologic irAEs associated with ipilimumab and nivolumab have been published [12]. Management guidelines encourage early use of high-dose steroids and discontinuation of immuno-oncologic agents for grade 2 events; while recommending specialist neurologic input and intravenous immunoglobulin (IVIG) for grade 3-4 events [13]. Unlike in colitis wherein the role of TNF- $\alpha$ inhibitors is clear $[14,15]$, the role of these agents in the management of grade 3/4 neurologic irAEs refractory to steroids and IVIG is unknown.

In this report, we describe two patients with melanoma who received ipilimumab in the adjuvant setting and developed ipilimumab-related neurologic irAEs. The first patient developed a meningoencephalomyelitis that relapsed despite high dose steroids and IVIG, but subsequently responded to a course of infliximab. The second patient developed an acute inflammatory demyelinating polyneuropathy (AIDP) that responded to high dose steroids. In this report, we highlight the importance of symptom recognition and appropriate diagnostic evaluation to early diagnosis of unusual neurologic irAEs permitting institution of appropriate immunosuppressive therapy.

\section{Case presentation \\ Patient 1}

A 39-year-old Caucasian female was diagnosed with nodular melanoma of the right upper back following a biopsy. Wide local excision (WLE) and right axillary sentinel lymph node (SLN) biopsy were performed; although WLE was negative, SLN was positive for multiple foci of residual melanoma in two LN. Completion lymph node dissection (CLND) was negative for any involvement of 19 LN. She was staged with IIIA (T3aN2aMx) disease and offered adjuvant therapy with ipilimumab based on the EORTC 18071 data [16]. Between January 4, 2017 and February 24, 2017, she received 3 doses of ipilimumab $10 \mathrm{mg} / \mathrm{kg}$ IV. Prior to fourth induction dose of ipilimumab, she developed flu-like symptoms and persistent headaches although no neurologic deficits were noted. Magnetic resonance imaging (MRI) of the brain and spine showed mild pituitary enlargement and leptomeningeal enhancement with a nodular focus of enhancement in the right internal auditory canal (Fig. 1a and b). On lumbar puncture, opening pressures were elevated ( $>30 \mathrm{~mm}$ water) while cerebrospinal fluid (CSF) examination was negative for any malignant cells but showed lymphocytic pleocytosis. Extensive testing excluded infectious and autoimmune etiologies; and other laboratory tests were consistent with central hypothyroidism and adrenal insufficiency. She was diagnosed with ipilimumab-related meningoencephalomyelitis, and treated with methylprednisolone $1.0 \mathrm{mg} / \mathrm{kg}$ daily with rapid improvement

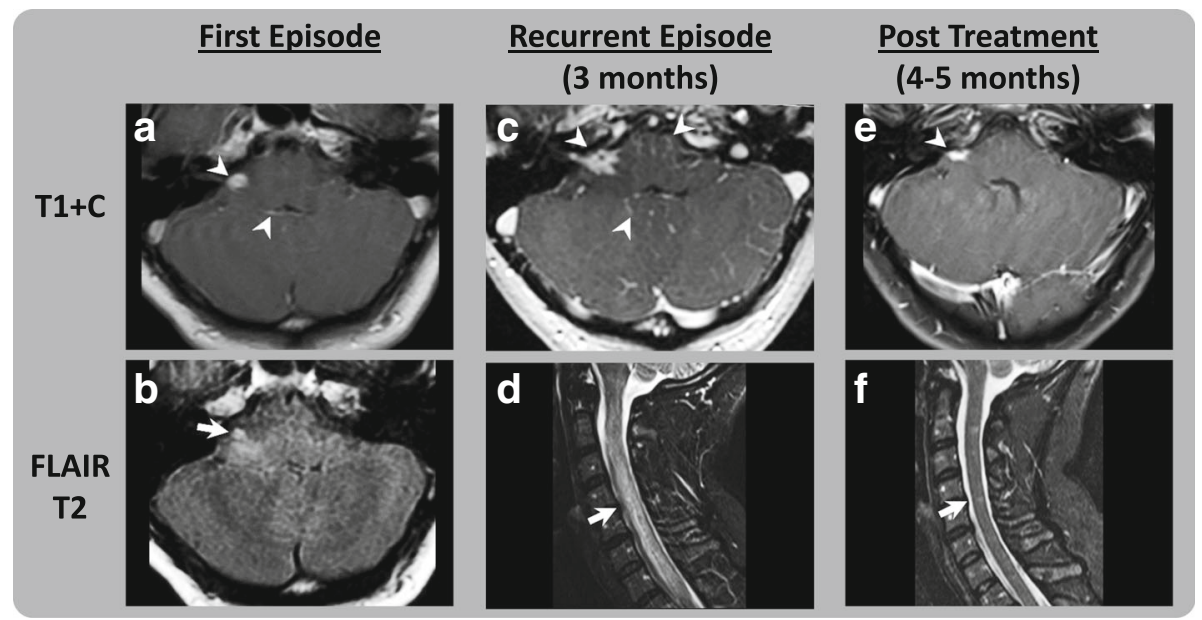

Fig. 1 Gadolinium Enhanced MRI Brain and Spine, Images of Patient 1. First Episode (a and b): Abnormal nodular leptomeningeal enhancement, most notably at the R-cerebellar pontine angle (CPA) and $4^{\text {th }}$ ventricle (arrowhead). Lack of FLAIR suppression at the basal cisterns is also most prominent at the R-CPA. There is associated parenchymal edema that extends into the right brachium pontis (arrow). Recurrent Episode (c and d): Worsening leptomeningeal enhancement, prominently involving the R-internal auditory canal (arrowhead). Holocord T2 signal hyperintensity consistent with edema resulting in complete effacement of the CSF space (arrow). Post-treatment (e and $\mathbf{f}$ ): Mild residual abnormal nodular enhancement at the right IAC (arrowhead) and near complete normalization of the cord diameter. There is only trace residual abnormal signal (arrow) 
and discharged on an oral prednisone taper. As an outpatient, prednisone was gradually tapered over an eight-week period till she was on physiologic doses of hydrocortisone. She was also treated with levothyroxine $100 \mathrm{mcg}$ daily for ipilimumab-related hypothyroidism. Ipilimumab adjuvant therapy was permanently discontinued.

Approximately 3 months following initial admission, she developed lower extremity weakness and fecal and urinary incontinence. MRI imaging of the brain and spine showed recurrent leptomeningeal and cranial nerve enhancement; diffuse lack of FLAIR suppression of CSF signal; and expansion of the cervical cord and T2 hyper-intense cord signal alteration with abnormal patchy intramedullary enhancement (Fig. 1c and d). CSF studies documented recurrent lymphocytic pleocytosis while serological studies were negative for autoimmune etiologies including paraneoplastic syndromes. Her CSF protein was elevated to $120 \mathrm{mg} / \mathrm{dL}$ (range 15-45 mg/dL). Imaging was negative for any evidence of recurrent malignancy. Per American Society of Clinical Oncology (ASCO) clinical practice guidelines, IVIG $1 \mathrm{~g} / \mathrm{kg}$ and methylprednisolone $1.0 \mathrm{mg} / \mathrm{kg}$ IV daily were administered for 5 days albeit with scant improvement. In the setting of IVIG/steroid refractory ipilimumab-related meningoencephalomyelitis, a trial of infliximab was offered. Infliximab $5 \mathrm{mg} / \mathrm{kg}$ IV was administered while prednisone $1 \mathrm{mg} / \mathrm{kg}$ daily was continued with improvement in neurologic function [17]. Two days after first dose of infliximab, she was able to ambulate with support although she continued to have urinary retention. Subsequently, she was discharged for spinal cord rehabilitation. Infliximab $5 \mathrm{mg} /$ $\mathrm{kg}$ was continued for a further two doses at two and 4 weeks following initial administration. Prednisone was continued at $60 \mathrm{mg}$ daily for 2 months then tapered gradually by $10 \mathrm{mg}$ every 2 weeks over a 3-month period after which she was transitioned to physiologic replacement doses of hydrocortisone on which she remains indefinitely. Her headaches and neurologic symptoms have improved substantially with near complete neurologic recovery including continence except mild spasticity in lower extremities. Repeat MRI imaging of the brain and spine documented progressively decreased leptomeningeal enhancement and eventual normalization of T2 signal (Fig. 1e and f). Restaging scans remain negative for recurrent melanoma.

\section{Patient 2}

A 55 year-old male was diagnosed with stage IIIB (T4bN1aMx) superficial spreading melanoma of the right distal thigh after initial surgery, right inguinal SLN biopsy and right inguinal CLND. He was treated with ipilimumab $10 \mathrm{mg} / \mathrm{kg}$ IV every 3 weeks and received 2 doses. Following second dose, he developed parasthesias in distal lower extremities bilaterally that subsequently ascended to involve proximal lower extremities, then upper extremities along with weakness and loss of deep tendon reflexes. No bladder or bowel incontinence and/ or difficulties with speech were observed. MRI brain and whole spine revealed abnormal enhancement involving the bilateral 5th, 7th and 8th cranial nerves, cauda

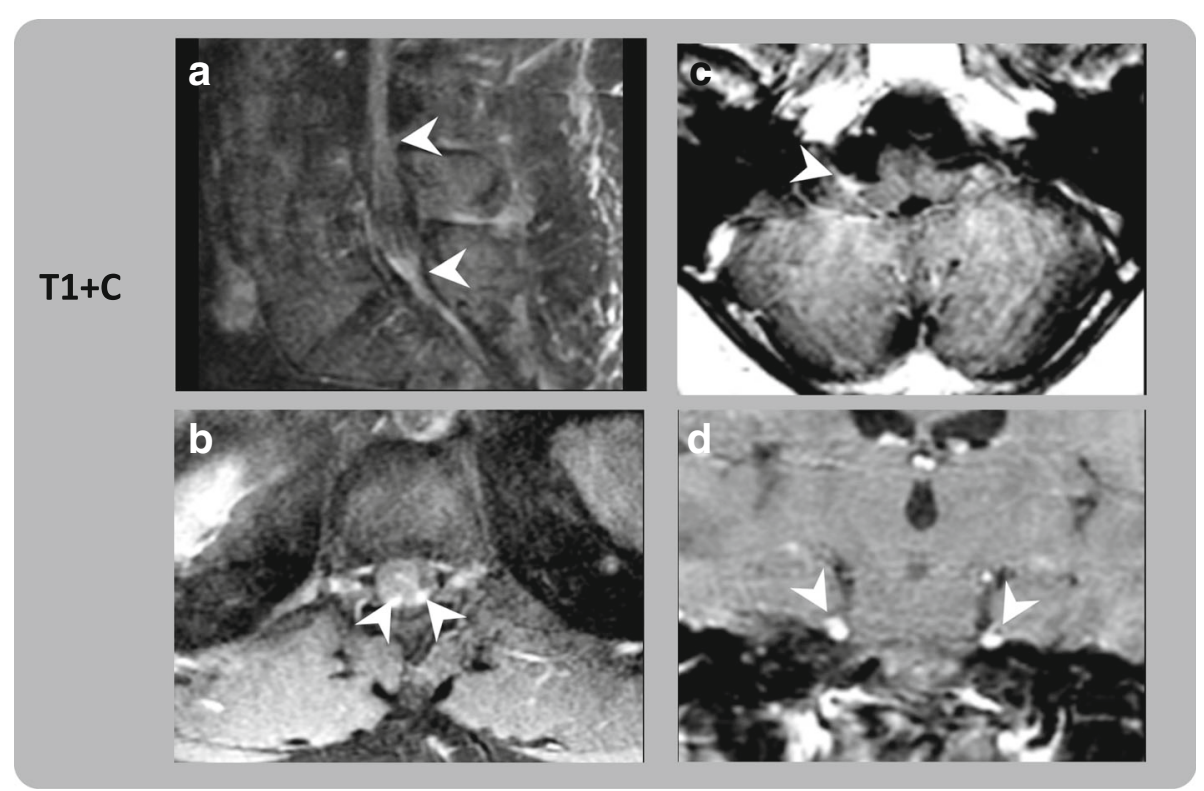

Fig. 2 Gadolinium Enhanced MRI Brain and Spine, Images of Patient 2. Post-contrast MR images demonstrate abnormal leptomeningeal enhancement throughout the CNS. (a) abnormal smooth thickening and enhancement of the cauda equina and nerve roots (arrowhead). (b) abnormal smooth thickening of the nerve roots at the thoracolumbar junction (arrowhead). (c) abnormal enhancement of the $7^{\text {th }}$ cranial nerve (arrowhead). (d) abnormal enhancement of both $5^{\text {th }}$ cranial nerves seen in a coronal plane (arrowhead) 
equina nerve roots as well as the conus surface and peripheral nerves at the thoracolumbar junction (Fig. 2a and d). CSF studies were negative for malignant cells but showed lymphocytic pleocytosis. CSF protein was elevated to $175 \mathrm{mg} / \mathrm{dL}$ (range 15-45 mg/dL). An extensive evaluation for autoimmune and paraneoplastic etiologies was negative although laboratory studies revealed concomitant adrenal insufficiency. Electromyography showed electrophysiologic evidence consistent with a generalized, sensory and motor, length-dependent, predominantly axonal, peripheral polyneuropathy without definite electrophysiologic evidence of a presynaptic neuromuscular junction transmission disorder or proximal myopathy. He was diagnosed with ipilimumabrelated acute inflammatory demyelinating polyneuropathy (AIDP).

Methylprednisolone $1.5 \mathrm{mg} / \mathrm{kg}$ twice daily was initiated with rapid improvement in motor symptoms in hands and lower extremities after 2 days. Steroids were tapered slowly from prednisone $1 \mathrm{mg} / \mathrm{kg}$ by decreasing by $10 \mathrm{mg}$ weekly over a 12 -week period with gradual recovery. Towards the end of the taper, prednisone was transitioned to physiologic doses of hydrocortisone to treat ipilimumab-related adrenal insufficiency. Although weakness resolved completely, he continued to have minimal parasthesias in upper extremities and lower extremities in a glove-and-stocking distribution. Approximately 4 months after initial ipilimumab therapy, he developed hypothyroidism for which he was treated with levothyroxine $125 \mathrm{mcg}$ daily. Systemic imaging studies remain negative for recurrent disease.

\section{Discussion}

Ipilimumab-associated neurological irAEs span diverse entities including severe meningo-radiculo-neuritis [18], reversible splenial lesions $[19,20]$, hypophysitis [21], meningitis [21], acute cerebellitis [22], Guillain-Barre syndrome [21, 23], myasthenia gravis [24-27], bilateral phrenic nerve paralysis [28], Bell's palsy [28], chronic inflammatory demyelinating polyneuropathy (CIDP) [29], transverse myelitis [29], encephalitis [30], necrotic myelopathy [31], and partial motor convulsive status (epilepsia partialis continua) [32]. Herein, we report two cases of ipilimumab-associated neurological irAEs manifesting as meningoencephalomyelitis and AIDP respectively. Although ipilimumab-related meningoencephalomyelitis and AIDP have both been reported previously, this report describes two patients with diverse manifestations including a relapse following initial immunosuppression requiring infliximab for resolution. Both patients also developed autoimmune thyroiditis and adrenal insufficiency in addition to neurological irAEs, suggesting that several immune-related adverse events including neurological manifestations can cluster. A review of previously published reports of ipiliumumab-associated neurological irAEs suggests that neurological irAEs occur both in isolation and with other irAEs with no consistent pattern linking the two (Table 1).

The tumor necrosis factor alpha (TNF- $\alpha$ ) inhibitor infliximab has a clear role in the management of steroid-refractory immune-related colitis [15, 33, 34], however its role in the treatment of neurologic irAE is unknown. In a prior report, infliximab was administered to treat severe ipilimumab-induced peripheral neuropathy that developed after two doses of adjuvant ipilimumab administered in the setting of stage III melanoma [35]. However, irAE was refractory to steroids, IVIG and infliximab and ultimately required tacrolimus for resolution. In another case report, infliximab ( $5 \mathrm{mg} / \mathrm{kg}$ IV) was given monthly for ipilimumab-induced necrotic myelopathy after lack of response to daily prednisone $(1 \mathrm{mg} / \mathrm{kg})$ for 2 weeks [31]. Four weeks after infliximab therapy, she is still wheelchair bound with painful paresthesia.

Table 1 Case Reports/Series of Ipilimumab-Associated Neurological irAEs

\begin{tabular}{|c|c|c|}
\hline Case reports/series & $\begin{array}{l}\text { Neurological } \\
\text { manifestations }\end{array}$ & $\begin{array}{l}\text { Presence of } \\
\text { additional irAEs }\end{array}$ \\
\hline $\begin{array}{l}\text { Wilgenhof S, et al. } \\
\text { (2011) [23] }\end{array}$ & $\begin{array}{l}\text { Guillain-Barré } \\
\text { Syndrome }\end{array}$ & None reported \\
\hline $\begin{array}{l}\text { Bompaire F, et al. } \\
(2012)[18]\end{array}$ & $\begin{array}{l}\text { Meningo-radiculo- } \\
\text { neuritis }\end{array}$ & None reported \\
\hline $\begin{array}{l}\text { Bot l, et al. } \\
(2013)[21] \\
(n=3)\end{array}$ & $\begin{array}{l}\text { Hypophysitis } \\
\text { Meningitis } \\
\text { Guillain-Barré } \\
\text { syndrome }\end{array}$ & \\
\hline $\begin{array}{l}\text { Liao B. et al. } \\
(2014)[21] \\
(n=3)\end{array}$ & $\begin{array}{l}\text { CIDP } \\
\text { Transverse myelitis } \\
\text { Myasthenia } \\
\text { gravis-type } \\
\text { syndrome }\end{array}$ & None reported \\
\hline $\begin{array}{l}\text { Johnson DB, et al. } \\
\text { (2015) [24] }\end{array}$ & Myasthenia gravis & $\begin{array}{l}\text { Thyroiditis } \\
\text { Dermatitis }\end{array}$ \\
\hline $\begin{array}{l}\text { Thaipisuttikul I, et al. } \\
\text { (2015) [35] }\end{array}$ & $\begin{array}{l}\text { Peripheral } \\
\text { neuropathy }\end{array}$ & None reported \\
\hline $\begin{array}{l}\text { Abdallah AO, et al. } \\
\text { (2016) [31] }\end{array}$ & $\begin{array}{l}\text { Necrotic } \\
\text { myelopathy }\end{array}$ & None reported \\
\hline $\begin{array}{l}\text { Chen JH, et al. } \\
\text { (2017) [27] }\end{array}$ & $\begin{array}{l}\text { Myasthenia } \\
\text { gravis }\end{array}$ & $\begin{array}{l}\text { Myositis } \\
\text { Polyneuropathy } \\
\text { Hepatitis }\end{array}$ \\
\hline $\begin{array}{l}\text { Derle E, et al. } \\
\text { (2018) [25] }\end{array}$ & Myasthenia gravis & None reported \\
\hline $\begin{array}{l}\text { Montes V, et al. } \\
\text { (2018) [26] }\end{array}$ & Myasthenia gravis & None reported \\
\hline $\begin{array}{l}\text { Naito T, et al. } \\
(2018)[22]\end{array}$ & Acute cerebellitis & None reported \\
\hline $\begin{array}{l}\text { Ruff MW, et al. } \\
(2018)[28] \\
(n=2)\end{array}$ & $\begin{array}{l}\text { Bilateral phrenic } \\
\text { nerve paralysis } \\
\text { Bell's palsy }\end{array}$ & Pan-hypopituitarism \\
\hline
\end{tabular}


Table 2 HLA Typing Results from Patients 1 and 2

\begin{tabular}{|c|c|c|c|c|c|c|c|c|}
\hline HLA Class I & A & $B$ & Bw & $C$ & $C w$ & & & \\
\hline \multirow[t]{2}{*}{ Patient 1} & $24: X X$ & 07:XX & $06: X X$ & $04: X X$ & $04: X X$ & & & \\
\hline & 29:XX & $35: X X$ & $06: X X$ & 07:XX & $07: X X$ & & & \\
\hline \multirow[t]{2}{*}{ Patient 2} & 11:01 & 27:05 & $04: X X$ & 01:02 & & & & \\
\hline & 24:02 & $35: 01$ & $06: X X$ & 04:01 & & & & \\
\hline HLA Class II & DRB1 & DRB3 & DRB4 & DRB5 & DQB1 & DQA1 & DPB1 & DRw \\
\hline \multirow[t]{2}{*}{ Patient 1} & 04:XX & & 01:XX & $01: X X$ & $03: X X$ & & & $53: X X$ \\
\hline & 15:XX & & & & $05: X X$ & & & 51:XX \\
\hline \multirow[t]{2}{*}{ Patient 2} & 01:01 & & 01:03 & & 03:02 & 01:01 & 03:01 & \\
\hline & 04:04 & & $53: X X$ & & 05:01 & 03:01 & 14:01 & \\
\hline
\end{tabular}

Exact mechanisms underlying CTLA-4 inhibitor toxicity remain unclear, although multiple hypotheses including loss of peripheral tolerogenic mechanisms via T-regulatory cell depletion [36, 37] and atypical expression of CTLA-4 on non-immune cells [11] have been advanced. Given links between CTLA-4 gene polymorphisms (in particular CTLA-4 59A > G variant) and incidence of autoimmune diseases, it is possible that CTLA-4 gene polymorphisms may mediate a certain fraction of irAEs related to CTLA-4 inhibition. Expression of certain major histocompatibility complex (MHC) or human leucocyte antigen (HLA) class-I and -II proteins (in particular HLA-DR) is associated with response to PD-1 blockade [38, 39]. However, whether certain MHC haplotypes have an increased risk of irAEs when treated with PD-1 and/or CTLA-4 inhibitor therapy is less well understood. The link between certain autoimmune diseases and particular HLA haplotypes is well known including DQB1 in GBS [39, 40]. Interestingly, both patients were haplo-identical at the HLA-DRB1*04, HLA-DRB4*01 and HLA-DQB1*03 alleles (Table 2). In the absence of larger studies with longer term follow up, the significance of this association is unknown.

The incidence of ipilimumab-related irAEs is clearly dose-related with a significantly greater incidence of irAEs at the $10 \mathrm{mg} / \mathrm{kg}$ dose compared to the $3 \mathrm{mg} / \mathrm{kg}$ dose $[5,6]$. However, the irAE incidence at the $10 \mathrm{mg} / \mathrm{kg}$ dose is similar in both adjuvant and metastatic disease settings $[6,16]$. This data suggests that irAE occurrence depends several upon host factors but may be agnostic of the disease burden. Recent reports have implicated interactions between the intestinal microbiome and $\mathrm{T}$ regulatory cells in mediating some aspect of ipilimumab related colitis but whether this extends to ipilimumab related neurologic irAEs is as yet unknown [41-43].

\section{Conclusions}

In this report, we describe two patients with melanoma who received ipilimumab in the adjuvant setting and developed distinct ipilimumab-related neurologic irAEs.
Early recognition and management of neurological irAEs is essential for maximizing clinical recovery and minimizing effect of drug-related toxicity. Although irAEs can occur at any stage of treatment, presentations within 4 months of treatment initiation is typical [44] as was seen in both these patients. Management of suspected neurological irAEs should incorporate early input of subspecialty neurologists attuned to fully characterize the neurological perturbation and to exclude leptomeningeal and/or paraneoplastic manifestations of cancer particularly in certain malignancies (melanoma, non-small cell lung cancer, etc.). Early initiation of immunosuppression with corticosteroids for grade 2 or greater events is critical; and steroids should be tapered slowly to avoid flares. The successful use of infliximab to treat a severe steroid/ IVIG-refractory neurologic irAE reported herein suggests that clinicians can consider the role of alternative immunosuppressive agents (mycophenolate, infliximab, etc.) to treat steroid-refractory neurological irAEs.

\section{Abbreviations}

AIDP: Acute inflammatory demyelinating polyneuropathy; CLND: Complete lymph node dissection; CSF: Cerebral spinal fluid; CTLA: Cytotoxic Tlymphocyte antigen-4; EORTC: European Organisation for Research and Treatment of Cancer; GBS: Guillain-Barre Syndrome; irAEs: Immune-related adverse effects; IV: intravenous; MRI: Magnetic resonance imaging; PD1: Programmed cell death protein 1; SLN: Sentinel lymph node; WLE: Wide local excision

Availability of data and materials

All materials and data are available to readers without qualifications upon request.

\section{Authors' contributions}

CG: Conceived of case report, wrote initial draft of manuscript and reviewed final manuscript. AE and TJ: Interpreted and created radiographic images; reviewed final manuscript. LC: Interpreted CSF studies including flow cytometry; reviewed final manuscript. VG and JD: Involved in decision making and care for both patients; reviewed final manuscript. DD: Involved in decision making and care for both patients; conceived of case report, revised initial draft and reviewed final manuscript. All authors read and approved the final manuscript.

\section{Ethics approval and consent to participate}

Both patients are participants in an existing University of Pittsburgh IRB-approved tissue collection and banking protocol, UPCI 96-099 (IRB number: IRB970186' University of Pittsburgh Cancer Institute Human Biological Sample and Nevus 
Image Banking and Analysis Protocol). A copy of their informed consent is available upon request.

\section{Consent for publication}

Informed written consent was obtained from patient for publication of this case. Consent is available upon request.

\section{Competing interests}

The authors declare that they have no competing interests.

\section{Publisher's Note}

Springer Nature remains neutral with regard to jurisdictional claims in published maps and institutional affiliations.

\section{Author details}

${ }^{1}$ Hillman Cancer Center, University of Pittsburgh Medical Center, 5150 Centre Ave, Pittsburgh, PA 15232, USA. ²Department of Radiology, University of Pittsburgh Medical Center, PUH Suite E204, 200 Lothrop Street, Pittsburgh, PA 15213, USA. ${ }^{3}$ Division of Hematopathology, Department of Radiology, University of Pittsburgh Medical Center, PUH Suite E204, 200 Lothrop Street, Pittsburgh, PA 15213, USA.

\section{Received: 22 March 2018 Accepted: 10 August 2018}

\section{Published online: 31 August 2018}

\section{References}

1. Krummel MF, Allison JP. CD28 and CTLA-4 have opposing effects on the response of T cells to stimulation. J Exp Med. 1995;182(2):459-65.

2. Leach DR, Krummel MF, Allison JP. Enhancement of antitumor immunity by CTLA-4 blockade. Science. 1996;271(5256):1734-6.

3. Selby MJ, et al. Preclinical development of Ipilimumab and Nivolumab combination immunotherapy: mouse tumor models, in vitro functional studies, and Cynomolgus macaque toxicology. PLoS One. 2016;11(9): e0161779.

4. Hodi FS, et al. Immunologic and clinical effects of antibody blockade of cytotoxic T lymphocyte-associated antigen 4 in previously vaccinated cancer patients. Proc Natl Acad Sci U S A. 2008;105(8):3005-10.

5. Hodi FS, et al. Improved survival with ipilimumab in patients with metastatic melanoma. N Engl J Med. 2010;363(8):711-23.

6. Robert $\mathrm{C}$, et al. Ipilimumab plus dacarbazine for previously untreated metastatic melanoma. N Engl J Med. 2011:364(26):2517-26.

7. Schadendorf $D$, et al. Pooled analysis of long-term survival data from phase II and phase III trials of Ipilimumab in Unresectable or metastatic melanoma. J Clin Oncol. 2015;33(17):1889-94.

8. Weber J. Review: anti-CTLA-4 antibody ipilimumab: case studies of clinical response and immune-related adverse events. Oncologist. 2007;12(7):864-72.

9. Larkin J, et al. Neurologic serious adverse events associated with Nivolumab plus Ipilimumab or Nivolumab alone in advanced melanoma, including a case series of encephalitis. Oncologist. 2017;22(6):709-18.

10. Ibrahim $R$, Berman D, de Pril $V$, et al. Ipilimumab safety profile: summary of findings from completed trials in advanced melanoma. J Clin Oncol. 2011; 29(15_Suppl):8583.

11. Iwama $\mathrm{S}$, et al. Pituitary expression of CTLA-4 mediates hypophysitis secondary to administration of CTLA-4 blocking antibody. Sci Transl Med. 2014;6(230):230ra45.

12. Postow MA, et al. Nivolumab and ipilimumab versus ipilimumab in untreated melanoma. N Engl J Med. 2015;372(21):2006-17.

13. Linardou $H$, Gogas $H$. Toxicity management of immunotherapy for patients with metastatic melanoma. Ann Transl Med. 2016;4(14):272.

14. Beck KE, et al. Enterocolitis in patients with cancer after antibody blockade of cytotoxic T-lymphocyte-associated antigen 4. J Clin Oncol. 2006;24(15): 2283-9.

15. Johnston $\mathrm{RL}$, et al. Cytotoxic T-lymphocyte-associated antigen 4 antibody-induced colitis and its management with infliximab. Dig Dis Sci. 2009;54(11):2538-40.

16. Eggermont AM, et al. Prolonged survival in stage III melanoma with Ipilimumab adjuvant therapy. N Engl J Med. 2016;375(19):1845-55.

17. Brahmer JR, et al. Management of Immune-Related Adverse Events in patients treated with immune checkpoint inhibitor therapy: American Society of Clinical Oncology clinical practice guideline. J Clin Oncol. 2018; 36(17):1714-68.
18. Bompaire $F$, et al. Severe meningo-radiculo-neuritis associated with ipilimumab. Investig New Drugs. 2012;30(6):2407-10.

19. Conry RM, Sullivan JC, Nabors LB 3rd. Ipilimumab-induced encephalopathy with a reversible splenial lesion. Cancer Immunol Res. 2015:3(6):598-601.

20. Williams TJ, et al. Association of Autoimmune Encephalitis with Combined Immune Checkpoint Inhibitor Treatment for metastatic Cancer. JAMA Neurol. 2016;73(8):928-33.

21. Bot I, et al. Neurological immune-related adverse events of ipilimumab. Pract Neurol. 2013;13(4):278-80.

22. Naito T, et al. Acute cerebellitis after administration of nivolumab and ipilimumab for small cell lung cancer. Neurol Sci. 2018; https://doi.org/10. 1007/s10072-018-3465-4

23. Wilgenhof S, Neyns B. Anti-CTLA-4 antibody-induced Guillain-Barre syndrome in a melanoma patient. Ann Oncol. 2011;22(4):991-3.

24. Johnson DB, et al. Myasthenia gravis induced by Ipilimumab in patients with metastatic melanoma. J Clin Oncol. 2015;33(33):e122-4.

25. Derle E, Benli S. Ipilimumab treatment associated with myasthenic crises and unfavorable disease course. Neurol Sci. 2018; https://doi.org/10.1007/ s10072-018-3471-6.

26. Montes $\mathrm{V}$, et al. Myasthenia gravis induced by Ipilimumab in a patient with metastatic melanoma. Front Neurol. 2018;9:150.

27. Chen $\mathrm{JH}$, et al. Coexisting myasthenia gravis, myositis, and polyneuropathy induced by ipilimumab and nivolumab in a patient with non-small-cell lung cancer: a case report and literature review. Medicine (Baltimore). 2017;96(50):e9262

28. Ruff MW, Mauermann ML. The Mayo Clinic experience with the neurological complications of the CTLA-4 inhibitor Ipilimumab. Neurologist. 2018;23(3):98-9.

29. Liao B, et al. Atypical neurological complications of ipilimumab therapy in patients with metastatic melanoma. Neuro-Oncology. 2014;16(4):589-93.

30. Graus F, et al. A clinical approach to diagnosis of autoimmune encephalitis. Lancet Neurol. 2016;15(4):391-404.

31. Abdallah $\mathrm{AO}$, et al. Ipilimumab-induced necrotic myelopathy in a patient with metastatic melanoma: a case report and review of literature. J Oncol Pharm Pract. 2016;22(3):537-42.

32. Mandel JJ, et al. Lambrolizumab induced central nervous system (CNS) toxicity. J Neurol Sci. 2014;344(1-2):229-31.

33. Arriola E, et al. Infliximab for IPILIMUMAB-related colitis-letter. Clin Cancer Res. 2015;21(24):5642-3.

34. Friedman CF, Proverbs-Singh TA, Postow MA. Treatment of the immunerelated adverse effects of immune checkpoint inhibitors: a review. JAMA Oncol. 2016;2(10):1346-53.

35. Thaipisuttikul I, Chapman P, Avila EK. Peripheral neuropathy associated with ipilimumab: a report of 2 cases. J Immunother. 2015;38(2):77-9.

36. Simpson $T R$, et al. Fc-dependent depletion of tumor-infiltrating regulatory $T$ cells co-defines the efficacy of anti-CTLA-4 therapy against melanoma. J Exp Med. 2013:210(9):1695-710.

37. Romano $\mathrm{E}$, et al. Ipilimumab-dependent cell-mediated cytotoxicity of regulatory T cells ex vivo by nonclassical monocytes in melanoma patients. Proc Natl Acad Sci U S A. 2015;112(19):6140-5.

38. Johnson DB, et al. Melanoma-specific MHC-II expression represents a tumour-autonomous phenotype and predicts response to anti-PD-1/PD-L1 therapy. Nat Commun. 2016:7:10582.

39. Jin PP, et al. Human leukocyte antigen DQB1 (HLA-DQB1) polymorphisms and the risk for Guillain-Barre syndrome: a systematic review and metaanalysis. PLoS One. 2015;10(7):e0131374.

40. Schirmer $L$, et al. Higher frequencies of $H L A$ DQB $1{ }^{*} 05: 01$ and antiglycosphingolipid antibodies in a cluster of severe Guillain-Barre syndrome. J Neurol. 2016;263(10):2105-13

41. Dubin $\mathrm{K}$, et al. Intestinal microbiome analyses identify melanoma patients at risk for checkpoint-blockade-induced colitis. Nat Commun. 2016;7:10391.

42. Chaput $\mathrm{N}$, et al. Baseline gut microbiota predicts clinical response and colitis in metastatic melanoma patients treated with ipilimumab. Ann Oncol. 2017; 28(6):1368-79.

43. Wang YN, et al. Controlling of glutamate release by neuregulin3 via inhibiting the assembly of the SNARE complex. Proc Natl Acad Sci U S A. 2018;115(10):2508-13.

44. Spain $L$, et al. Neurotoxicity from immune-checkpoint inhibition in the treatment of melanoma: a single Centre experience and review of the literature. Ann Oncol. 2017;28(2):377-85. 